\title{
Detection of cotton waterlogging stress based on hyperspectral images and convolutional neural network
}

\author{
Jing Zhao ${ }^{1,2}$, Fangjiang Pan ${ }^{1,2}$, Zhiming $\mathrm{Li}^{1,2}$, Yubin $\mathrm{Lan}^{1,2^{*}}$, Liqun $\mathrm{Lu}^{2,3^{*}}$, \\ Dongjian Yang ${ }^{1,2}$, Yuting Wen ${ }^{1,2}$ \\ (1. School of Agricultural Engineering and Food Science, Shandong University of Technology, Zibo 255000, Shandong, China; \\ 2. Sub-center of National Center for International Collaboration Research on Precision Agricultural Aviation Pesticides Spraying \\ Technology, Shandong University of Technology, Zibo 255000, Shandong, China; \\ 3. School of Traffic and vehicle engineering, Shandong University of Technology, Zibo 255000, Shandong, China)
}

\begin{abstract}
Waterlogging in the early stage of cotton will reduce the number of bolls and do harm to yield. Early detection of waterlogging will help farmers to adjust cotton management and save the loss. To evaluate the application of deep learning for the detection of early waterlogging, this study applied a convolutional neural network (CNN) to classify different durations of waterlogging stress $(0,2,4,6,8,10 \mathrm{~d})$ based on hyperspectral images (HSIs) of cotton leaves. An experiment was designed to simulate the situation of cotton under waterlogging stress and collect HSIs of visible and near-infrared (VNIR 450-950 nm) spectra with 126 bands $66 \mathrm{~d}$ after cotton sowing (66 DAS). It was found the spectral curve reflectance of waterlogging cotton was higher than that of non-waterlogging cotton. Especially near $550 \mathrm{~nm}$ and $750 \mathrm{~nm}$, and the spectral curve increased with durations of waterlogging stress and there were 'blue shift' phenomena for the position of the red edge of the spectra. The first principal components of HSIs after band randomly discarding and principal component analysis (PCA) were used to build a dataset. GoogLeNet Inception-v3 (GLNI-v3) and VGG-16 models were selected to detect cotton waterlogging stress with the dataset. The results showed that the average time for a round training for GLNI-v3 was $13.337 \mathrm{~s}$, with a classification accuracy of $96.95 \%$ and a loss value of 0.09431 . The average time for a round training for VGG-16 was $21.470 \mathrm{~s}$, with a classification accuracy of $97.00 \%$ and a loss value of 0.17912 . Though these two models had similar classification accuracy and loss value, GLNI-v3 achieved a high accuracy with fewer training iterations. The durations of waterlogging stress of cotton in a short-term can be detected by HSIs of cotton leaves and CNN models are suitable for the classification of HSIs, and this method can provide support for cotton yield estimation and loss assessment after waterlogging.
\end{abstract}

Keywords: cotton, waterlogging, hyperspectral image, convolutional neural network

DOI: $10.25165 /$ j.ijabe.20211402.6023

Citation: Zhao J, Pan F J, Li Z M, Lan Y B, Lu L Q, Yang D J, et al. Detection of cotton waterlogging stress based on hyperspectral images and convolutional neural network. Int J Agric \& Biol Eng, 2021; 14(2): 167-174.

\section{Introduction}

As the change of global climate, the frequency of extreme weather increases so that waterlogging events have become more common. Cotton is known to be poorly adapted to waterlogging which is one of the major problems that influences global cotton production $^{[1,2]}$. The root system of waterlogging cotton could not

Received date: 2020-07-14 Accepted date: 2020-12-03

Biographies: Jing Zhao, $\mathrm{PhD}$, Associate Professor, research interest: agricultural remote sensing and intelligent detection research, Email: zbceozj@163.com; Fangjiang Pan, Master candidate, research interest: agricultural remote sensing and intelligent detection research, Email: 181831794@163.com; Zhiming Li, Master, research interest: agricultural remote sensing and intelligent detection research, Email: 2295386984@qq.com; Dongjian Yang, Master candidate, research interest: agricultural remote sensing and intelligent detection research, Email: 694415363@qq.com; Yuting Wen, Master candidate, research interest: agricultural remote sensing and intelligent detection research, Email: 1256596123@qq.com.

*Corresponding author: Yubin Lan, $\mathrm{PhD}$, Professor, research interests: agricultural machinery. Shandong University of Technology, No. 266 Xincun West Road, Zhangdian District, Zibo 255000, Shandong, China. Email: ylan@sdut.edu.cn; Liqun Lu, PhD, Associate Professor, research interest: agricultural aviation technology and fluid transmission technology research. Shandong University of Technology, No. 266 Xincun West Road, Zhangdian District, Zibo 255000, Shandong, China. Email: luliqunustb@163.com. obtain the oxygen for aerobic metabolism and it has to turn to anaerobic metabolism which reduces root uptake of nitrogen and delays the growth of root ${ }^{[3]}$. Waterlogging reduces the number of bolls, which is associated with yield loss and lower radiation use efficiency causing the reduction of dry matter. Cotton with a high ridge has no significant reduction in yield with short-term waterlogging, such as $3 \mathrm{~d}^{[4]}$. Cotton is higher sensitivity to earlier waterlogging at squaring stages than that at flowering and boll-setting stages. Lint yield of cotton could be affected by waterlogging at squaring, flowering and boll-setting stages ${ }^{[5]}$. An early waterlogging event ( $65 \mathrm{~d}$ after cotton sowing, 65 DAS) makes radiation use efficiency remain low for the duration of cotton growth and waterlogging (66 DAS) of 3, 6, 9 and $12 \mathrm{~d}$ results in a $16 \%$ to $50 \%$ reduction in lint yield ${ }^{[6-8]}$. Early waterlogging reduces all nutrient concentrations in leaves as well ${ }^{[9]}$. Waterlogging and shade will impair leaf nitrogen acquisition, and the interactive effect of them is significant only when the level of waterlogging stress is light ${ }^{[10,11]}$.

A hyperspectral image (HSI) contains hundreds of narrow spectral bands from visible to near-infrared. Compared to RGB images or multispectral images, HSIs have better spectral resolution and record more spectral information of target. Hyperspectral imaging technology has been applied for plant disease and stress detection on leaf, single plant and canopy scale. 
The detection of leaves under biotic or water stress can be performed by HSIs and classifiers, such as quadratic discriminant analysis, neural networks, support vector machine, etc ${ }^{[12-14]}$. Vegetation indices, a subset of selected wavelengths and texture extracted from HSIs, are chosen as features for classification of healthy and diseased plants ${ }^{[15-18]}$. In the current studies of cotton, hyperspectral data was applied for the detection of foreign matter in $\operatorname{cotton}^{[19,20]}$, a subset of selected wavelengths were used for regression analysis to detect severity of pests ${ }^{[21,22]}$, and new indices of hyperspectral were combined to estimate the leaf water content $^{[23,24]}$.

The Yellow River Basin, Yangtze River Basin and Northwest Inland are the three major cotton producing regions in China ${ }^{[25]}$. Affected by monsoon, rainy season often occurs from June to September in Yellow River Basin and Yangtze River Basin, which may cause waterlogging of cotton. Except heavy rainfalls and floods, excessive irrigation can be a reason for waterlogging. Previous researches demonstrated that with various of waterlogging stress the reduction of yield was quite different ${ }^{[7,8]}$. Manual records cannot reflect the true days of waterlogging, which may lead to the mistaken estimation of yield after disaster. Convolutional neural network (CNN) model has shown the great ability of classification of plant disease and the CNN was applied for the detection of cotton waterlogging in this study. To evaluate the application of $\mathrm{CNN}$ for early waterlogging stress detection with hyperspectral data, an experiment was designed for cotton waterlogging treatment aiming to classify waterlogging stress in short-term with HSIs of cotton leaves. The objectives of this study were: (1) to obtain HSIs of waterlogging cotton, (2) to preprocess HSIs, (3) to compare spectral reflectance of cotton under waterlogging stress with that under normal condition, and (4) to perform classification of waterlogging stress with HSIs.

\section{Materials and methods}

\subsection{Design of waterlogging experiment}

The experiment started at the Experimental Station of Shandong Cotton Research Center, Linqing, Shandong, China $\left(36^{\circ} 61^{\prime} \mathrm{N}, 115^{\circ} 42^{\prime} \mathrm{E}\right)$ in the summer of 2019 . Linqing is a famous cotton-producing area because its soil and climate are ideal for cotton cultivation. The Experimental Station is located in the southern suburb of Linqing. It has a test area of $3.3 \mathrm{hm}^{2}$ with irrigation and drainage systems. The region is with a warm temperate monsoon climate and belongs to a hail-free zone, which represents the cotton cultivation environment in the Yellow River Basin.

In this study, the treatment of different waterlogging days was firstly designed, then the data collection and pretreatment were carried out. Finally, the data after pretreatment were analyzed. The overall flow chart is shown in Figure 1.

The waterlogging experiment was carried out in the bottomless earth pond, in which each plot was separated by concrete walls of $15 \mathrm{~cm}$ thick and $150 \mathrm{~cm}$ deep to prevent water loss. There were 12 plots and each had an area of $10.44 \mathrm{~m}^{2}(2.87 \mathrm{~m} \times 2.77 \mathrm{~m})$ for cotton planting. An electric rain shelter was installed above the earth pond. When the sensor on the rain shelter identified precipitation, it would turn on the motor to drive rain shelter to cover earth pond, aiming to prevent the interference of natural precipitation on the waterlogging experiment. When the rain stopped, the shelter would be automatically retracted. The earth pond and electric rain shelter are shown in Figure 2a.
Cotton was sown at a density of six plants $/ \mathrm{m}^{2}$ with four rows per plot on April 28, 2019, and the flower bell period was on July, 2019. There were three identical waterlogging treatment areas and one non-waterlogging control area. Each treatment area had three repeat plots (Figure 2b). Waterlogging treatment started from July 4, 2019 and lasted a total of $10 \mathrm{~d}$. Waterlogging treatment areas were supplied with water twice a day to ensure a 10 $\mathrm{cm}$ high water layer above the soil surface. The non-waterlogging control maintained normal irrigation according to local cotton planting experience.

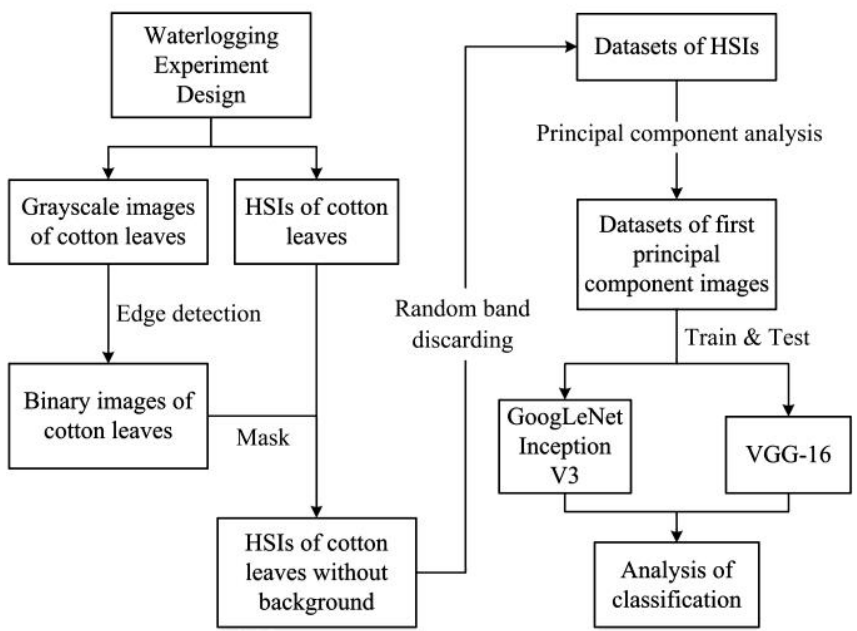

Figure 1 Flow chart of experiment and data analysis

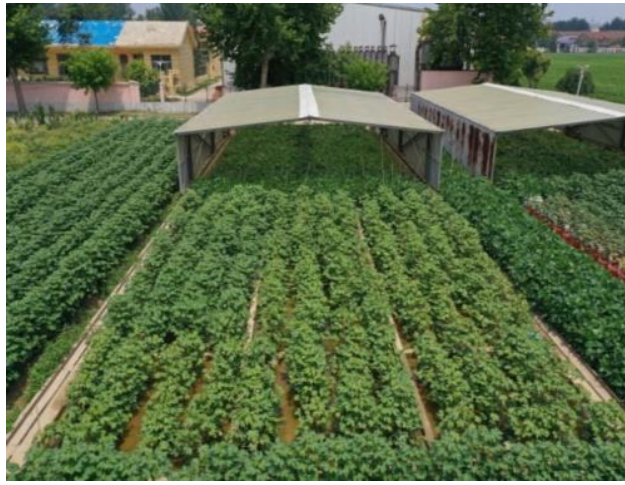

a. Earth pond and electric rain shelter

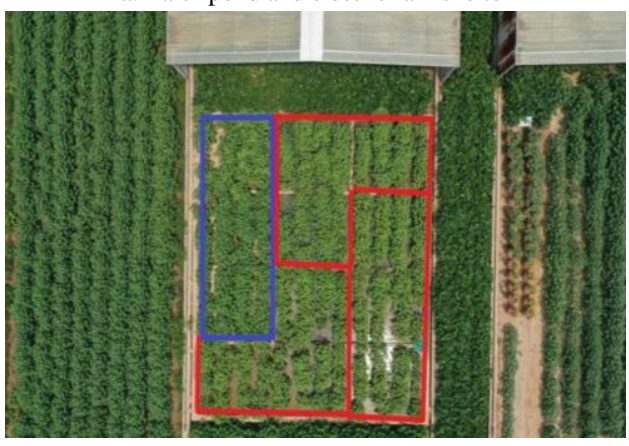

Note: red: waterlogging treatment; blue: control group

b. Areas of waterlogging and non-waterlogging

Figure 2 Introduction of the study area

\subsection{Collection of hyperspectral images}

HSIs were collected by a UHD-185 hyperspectral imaging camera (Cubert, Germany) 2, 4, 6, 8, $10 \mathrm{~d}$ after waterlogging treatment began, respectively. The hyperspectral imaging camera had a spectrum collection scope of 450-950 nm, spectral resolution of $4.0 \mathrm{~nm}$ and the image resolution was $1000 \times 1000$ pixels with 126 spectral channels. The software control system consisted of a high-performance laptop (Dell Inspiron 7559, i5-6300HQ, 
$2.30 \mathrm{GHz}$ CPU, 4 GB installed memory, 64-bit Windows 10 operating system) and Cube-Pilot Edelwei $\beta$ software. Illumination was provided by a halogen lamp of $100 \mathrm{~W}$ (Figure 3). Six fresh cotton leaf samples with no disease, insect pests, or damage were randomly collected from each plot, and a total of 90 HSIs of non-waterlogging control cotton leaves and 270 images of leaves with different waterlogging days were obtained for a total of five times (54 images every time). In order to reduce the influence of dark current and light on the quality of HSIs, the reference intensity of a standard white plate and dark environment were acquired so that the original reflectance intensity of HSIs could be converted to relative reflectance using Equation (1):

$$
R=\frac{I_{s}-I_{d}}{I_{w}-I_{d}} \times 100 \%
$$

where, $I_{s}$ is pixel value of origin HIS; $I_{w}$ is reflectance of standard white plate; $I_{d}$ is reflectance of dark environment; $R$ is relative reflectance, $\%$.

The HSIs were saved in grayscale with a ".cab" suffix. Cube-Pilot software was used to read files with ".cub" suffix, and saved the data to TIFF files.

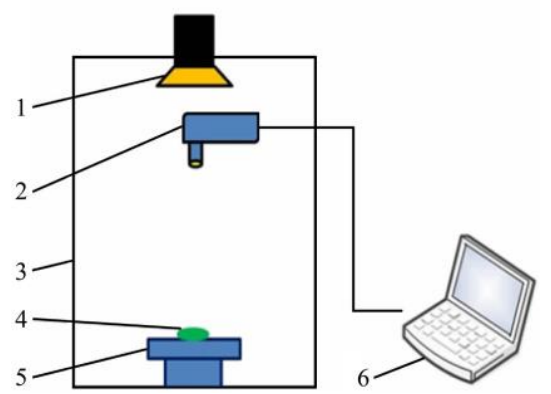

$\begin{array}{lll}\text { 1. Lighting assembly } 2 \text {. Hyperspectral imaging camera 3. Dark box } & \end{array}$ 4. Leaf samples 5. Linear translation stage 6. Computer a. Hyperspectral image acquisition system

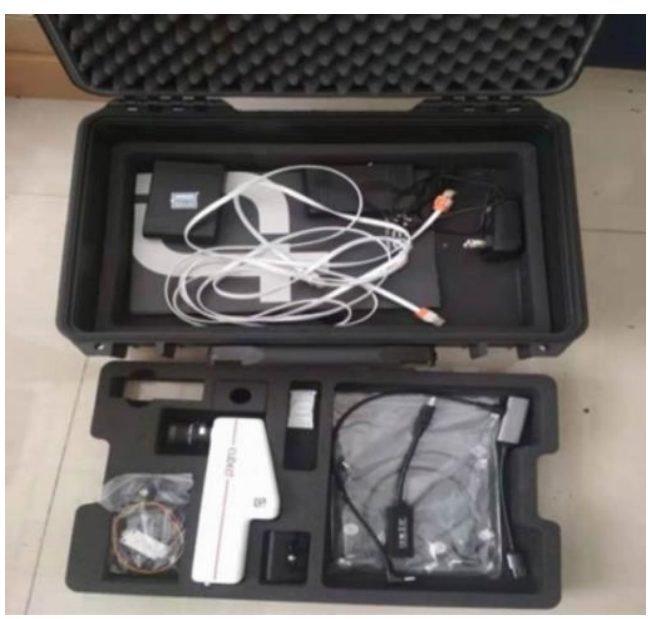

b. Hyperspectral imaging camera

Figure 3 Data collection devices

\subsection{Extraction of spectral data}

To analyze the effect of waterlogging stress on cotton leaf spectrum, ENVI 5.3 software was used to select region of interests (ROIs) to extract the spectral reflectance of cotton leaves. Six ROIs with $100 \times 100$ pixels were selected in every hyperspectral image. Mean spectral reflectance of different days of waterlogging treatment were calculated. Due to the influence of the environment and instruments, a certain amount of noise appeared in the raw data of spectral reflectance. Savitzky-Golay (SG) smoothing convolution method was applied to reduce the influence of noise in the spectral reflectance curve $e^{[26,27]}$. And the baseline drift of original spectral data was solved by multiple scattering correction (MSC).

\subsection{Removal of image background}

The images (Figure 4a) collected by the hyperspectral imaging camera contain background and the target leaves. A mask file was used to remove the background of images. Grayscale images of cotton leaves (Figure 4b) were processed by OpenCV under Python 3.7 version compilation environment. The Canny operator was selected to perform a convolution operation on the grayscale images to extract the edge contour of cotton leaves ${ }^{[28]}$. After Canny operation, binary images of leaves edge (Figure 4c) were obtained, but some edges of binary images were not closed. Morphological process was applied to all images to get a completed contour of leaves. It is a method of image processing that mainly includes two basic operations of corrosion and dilation and two combined operations of open and close operations. In order to fill the gaps in the contour, close operation was applied to perform dilation and then corrosion operation (Figure 4d). The leaf was finally shown with pixel value of 1 and the other area displayed with pixel value of 0 , so that the mask files (Figure 4e) were completed. The HSIs were processed with mask files to remove background by Python 3.7 (Figure 4f).

\subsection{Principal component analysis of images}

It is necessary to concentrate the effective information from all bands of HSIs to fewer conversion bands, which can maximize characterization of the original data information, because of the redundant information and correlation of HSIs bands. Therefore, principal component analysis (PCA) was used to perform dimensionality reduction on HSIs in this study. PCA maps data from the original coordinate system to a new orthogonal coordinate system through matrix transformation ${ }^{[29,30]}$. The first coordinate axis selects the direction with the largest variance in the original data. The second coordinate axis selects the direction that is orthogonal to the first coordinate axis and has the second largest variance. The selection of coordinate axis will be repeated until the number of new coordinate axes is equal to the dimension of the original data. The first principal component contains the largest variance information, followed by the second and third principal component, and the last few principal components have the least information.

The first principal component acquired by PCA process included more than $91 \%$ information of the original HSI. This study replaced the original HSIs with the images of the first principal component after PCA, which were used as samples for CNN training. The process of PCA and the selection of first principal component were completed using ENVI Tasks function through the ENVI Py Engine library with Python.

\subsection{Convolutional neural network}

In this study, CNN was used as the classifier for the classification of cotton leaves with different waterlogging stress. The basic blocks of CNN included convolution layer, activation layer, pooling layer and full connection layer ${ }^{[31-33]}$.

The convolutional layer is the core cornerstone of CNN, and the filter kernel in the convolution layer performs convolution operation on the image. The size of filter kernel is $n \times n$ ( $n$ is odd), which can be adjusted according to the needs. Filter kernel is slid over the image and dot product is calculated between the corresponding position of image and filter kernel. The weights in filter kernel are constantly optimized and updated by the back-propagation algorithm in the training process of CNN. 


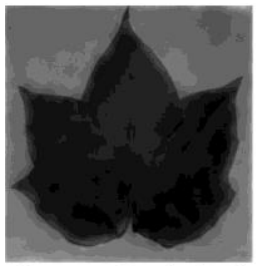

Non-waterlogging leaf

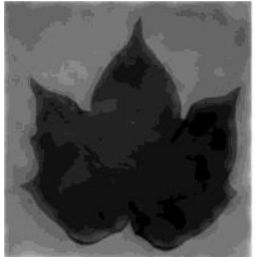
6 days
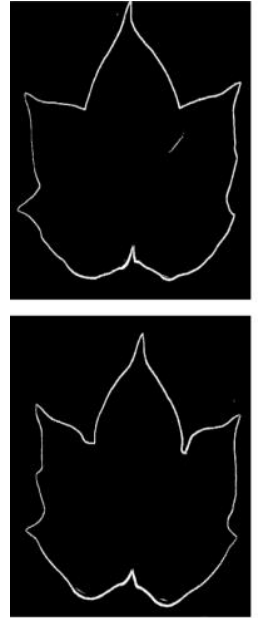

c. Leaf contour extracted by Canny operator
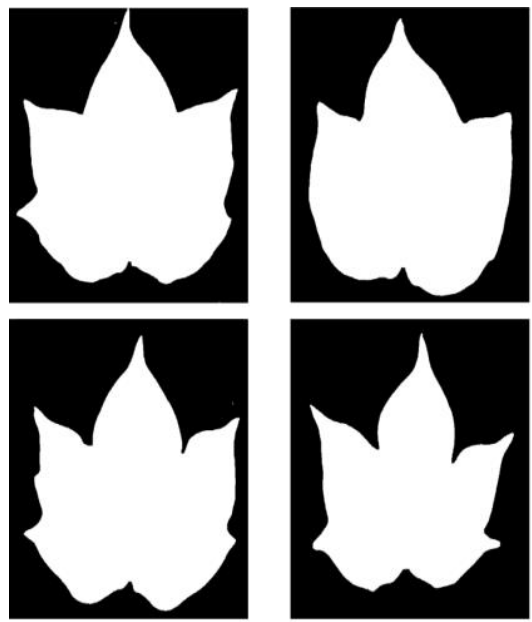

e. Mask of cotton leaves

a. Cotton leaf images of $454 \mathrm{~nm}$

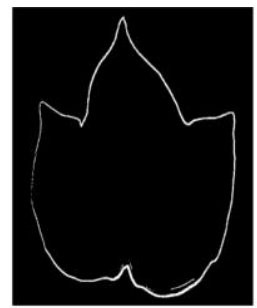

Leaf with waterlogging

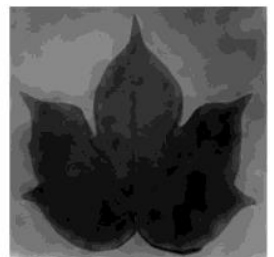

Leaf with waterlogging 4 days

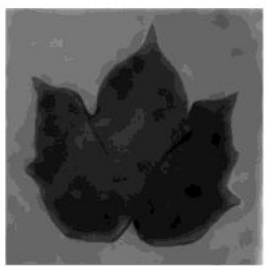
10 days
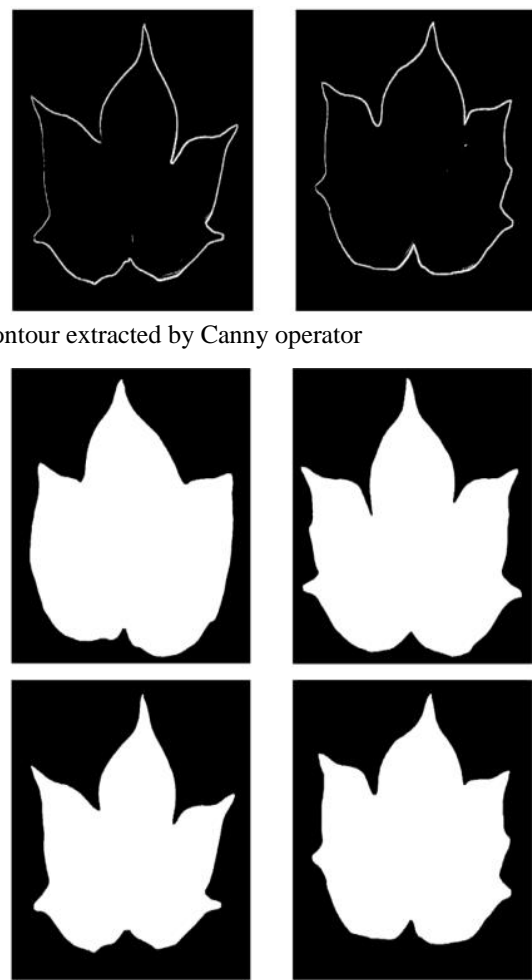

Figure 4 Different processing results

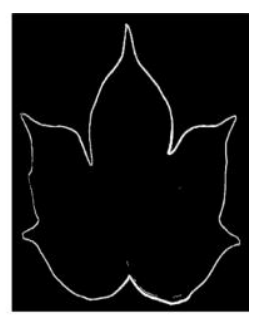

Leaf with waterlogging

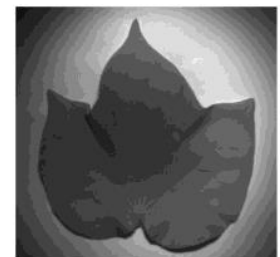

Leaf with waterlogging 2 days

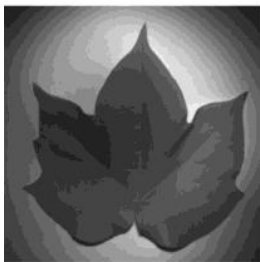

Leaf with waterlogging 6 days

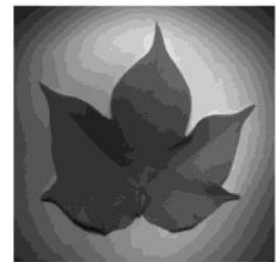

Leaf with waterlogging 8 days

b. Grayscale images of cotton leaf
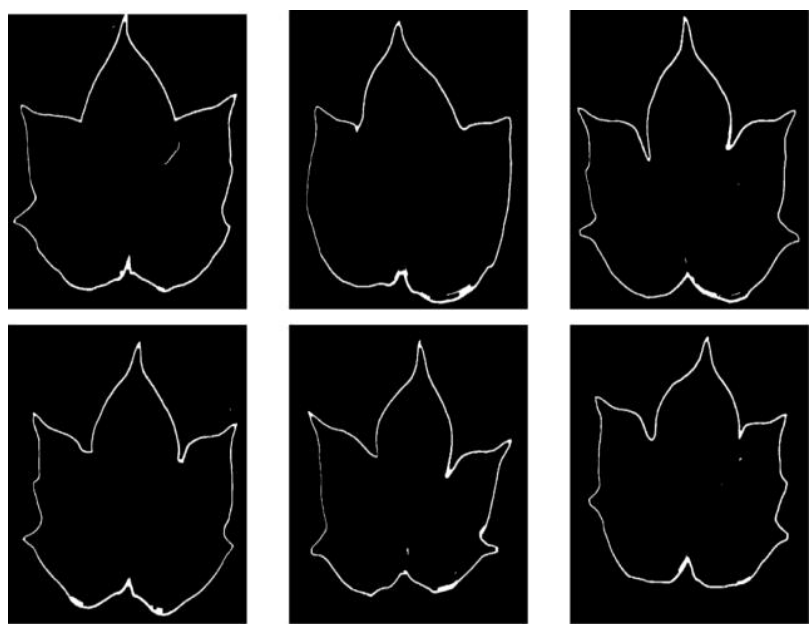

d. Leaf contour after close operation
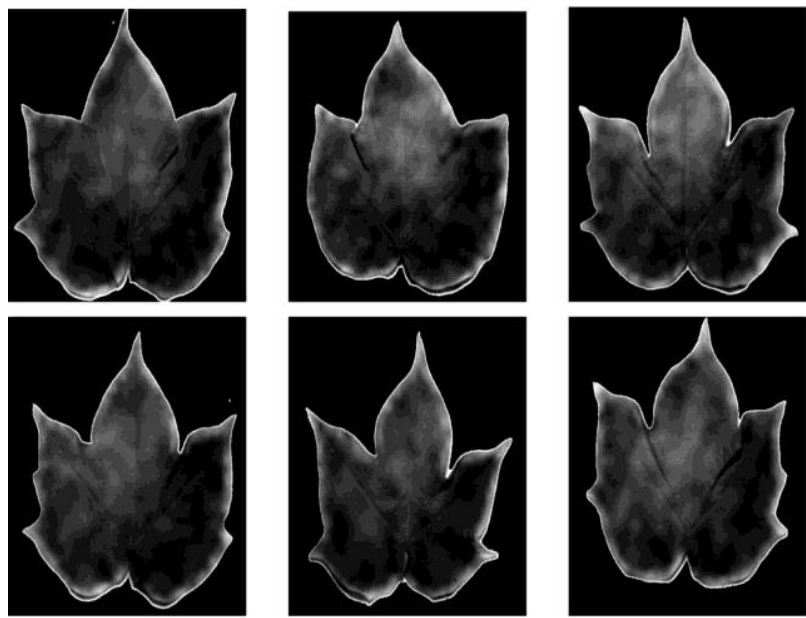

f. HSIs after masking

Leaf with waterlogging 10 days
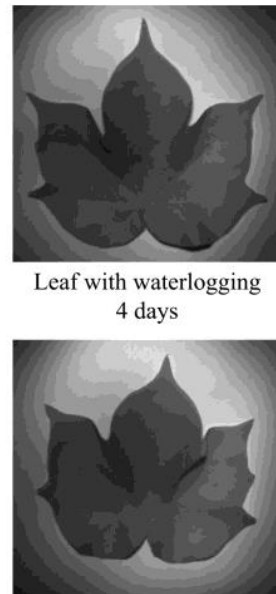

Leaf with waterlogging$$
\text { (1) }
$$

The input is mapped to the output by nonlinear function in
ivation layer. There are some nonlinear activation functions activation layer. There are some nonlinear activation functions
used in deep learning, such as sigmoid, tanh, ReLU, etc. Different activation functions are selected based on the learning task of neural network. The rectified linear unit (ReLU) is applied widely in $\mathrm{CNN}$, it can convert the input of negative value to 0 while keeping the input of non-negative value unchanged, which is convenient for model calculation. The ReLU function is defined as Equation (2):

$$
f(x)=\max (0, x)
$$

The pooling layer performs downsampling on the feature maps the sizes of feature maps and the weight parameters of the subsequent network. The general size of the pooling layer is $2 \times 2$, and the most commonly used calculation method is maximum pooling to extract maximum value of feature maps.

The nodes between fully connected layers no longer use local connections, but perform the fully connected method to increase the connection weight and expand the calculation of fully connected layer. The features extracted by $\mathrm{CNN}$ from input outputted by convolution layer and activation layer, which reduces 
images through layer-by-layer convolution correspond to the image labels, and the prediction results of image detection are given.

This study applied 2 classical CNN model (GLNI-v3 ${ }^{[34]}$ and VGG- $16^{[35]}$ ) as classifiers. The training and verification of the 2 models were conducted in Python 3.7 by Keras library. It is a Python artificial neural network library, which can be used as the application interface of Tensorflow, Microsoft-CNTK and Theano neural network library to conduct the structural design, parameter debugging, effect evaluation, model application and parameter visualization of deep learning model.

\subsection{Dataset of CNN}

During the waterlogging experiment, a total of 360 HSIs were collected. The training of CNN usually requires a large number of data, but the current manual collection of HSI is difficult to provide sufficient data support. In order to prevent overfitting caused by insufficient data, it is necessary to use data enhancement techniques to expand the amount of trainable data for a limited data set. The operations of flip, rotation, scale, shift, etc., to original HSIs can be regarded as adding noise to images. It can enhance the diversity of dataset and improve the generalization ability and robustness of CNN.

Due to the data redundancy between HSIs' bands, when a certain hyperspectral band is randomly discarded, the loss information can be supplemented by other related bands without causing major modifications to the original image. Meanwhile, randomly discarding a certain band from images produces difference between original and new images, which makes the data more diverse. In this study, band randomly discarding strategy was applied to every HSI. A round of band randomly discarding doubled the number of images, and the images were performed 5 times for waterlogging cotton and 4 times for non-waterlogging cotton. A total of 10080 images were collected. After the application, all the images were performed by PCA and the images of first principal component were reserved. 10080 images were divided into training images and verification images with the ratio of 3:1. There were 1296 training images and 432 verification images for each different waterlogging stress, as well as 1080 training images and 360 verification images for non-waterlogging treatment.

The training images and the verification images were stored in the folders named "Train" and "Test", and were further allocated to subfolders named "after days of waterlogging treatment". When using Python 3.7 to train the convolutional neural network model, the images were imported in the form of an array, normalized and scaled to put into training tensors and verification tensors. The label corresponding to each image was obtained by extracting the name of the folder where the image was located, and was encoded by one-hot. Stochastic Gradient Descent (SGD) was selected for weight update in the convolutional neural network model, and the model learning rate was set to 0.01 . The training round epochs were set to 1000 . The models were trained by all images of the training set in a single round. The batch size was set to 32 so that 32 images were randomly selected and delivered into the models at the same time for training. 5 folds cross validation was applied to the training to make sure the stability of accuracy. Cross entropy was selected as the loss function to score the classification results. The cross entropy is defined as Equation (3):

$$
H(p, q)=\sum_{x} p(x) \log \left(\frac{1}{q(x)}\right)
$$

where, $p$ is the true value probability distribution; $q$ is the model prediction value probability distribution.

\section{Results and discussion}

\subsection{Variation of spectral curve with different days of waterlogging treatment}

The average spectral curves of leaves in different waterlogging treatment days can be seen in Figure 5. The shapes of cotton leaves' spectral curves in waterlogging treatment are approximately the same as those in the non-waterlogging control group. The spectral curve of non-waterlogging treatment has a low reflectance in the range of 450 to $500 \mathrm{~nm}$. The curve begins to rise after $500 \mathrm{~nm}$ and reaches a small peak of reflectance around $550 \mathrm{~nm}$ affected by chlorophyll reflection. From 600 to $700 \mathrm{~nm}$, affected by strong absorption of chlorophyll, the curve decreases and drops to the lowest point around $670 \mathrm{~nm}$ appearing a valley of reflectance. After that, the spectral curve in the range of 670 to $750 \mathrm{~nm}$ shows a rapid upward trend, and a peak platform of reflectance occurs after $750 \mathrm{~nm}$.

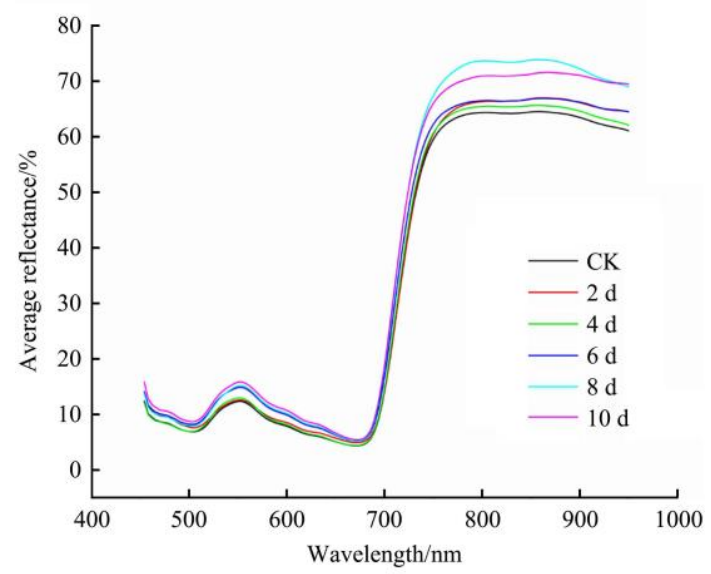

Figure 5 Original average spectral curves of cotton leaves under different waterlogging conditions

In Figure 6, it is observed that the reflectance of spectral curve with waterlogging treatment is higher than that with non-waterlogging spectral curve from 450 to $950 \mathrm{~nm}$. In the range of 500 to $550 \mathrm{~nm}$, the reflectance increases with the increase of the waterlogging days. At the peak of reflectance around $550 \mathrm{~nm}$, the curve of $10 \mathrm{~d}$ waterlogging treatment has the highest reflectance and the curve of $2 \mathrm{~d}$ waterlogging treatment has the lowest reflectance. In the range of 670 to $750 \mathrm{~nm}$ (red edge), the reflectance also increases with the increase of waterlogging days, the curve of $10 \mathrm{~d}$ waterlogging treatment is on far left side and the curve of $2 \mathrm{~d}$ waterlogging treatment is on far right side. There are 'blue shift' phenomena for the position of red edge wavebands. The reflectance in waterlogging treatment from 750 to $950 \mathrm{~nm}$ is quite different. The curve with $8 \mathrm{~d}$ waterlogging treatment has the highest reflectance.

In visible wavebands, due to the absorption of chlorophyll, there is a valley near $500 \mathrm{~nm}$ and $670 \mathrm{~nm}$ waveband, and a red edge is formed from $670 \mathrm{~nm}$ to $750 \mathrm{~nm}$. In the range from $500 \mathrm{~nm}$ to $600 \mathrm{~nm}$ waveband, a green peak is formed because of the reflection of chlorophyll. In near-infrared waveband, the spongy mesophyll of leaves makes a reflecting platform with high reflectance from $750 \mathrm{~nm}$ to $950 \mathrm{~nm}$ waveband. Previous studies ${ }^{[5,8]}$ have shown that, with the increased durations of waterlogging stress, the concentration of chlorophyll in leaves significantly reduces and photosynthesis of cotton decreases. Waterlogging has made the chlorophyll synthesis associated gene (GhLHCB) down-regulate so that chlorophyll synthesis rate decreases. And the concentration 
of $\mathrm{H}_{2} \mathrm{O}_{2}$ in tissues increases with the increase of waterlogging stress, which would cause damage to cell membranes under hypoxic conditions. These may be the main reason for the increased spectral reflectance of cotton under the durations of waterlogging stress.

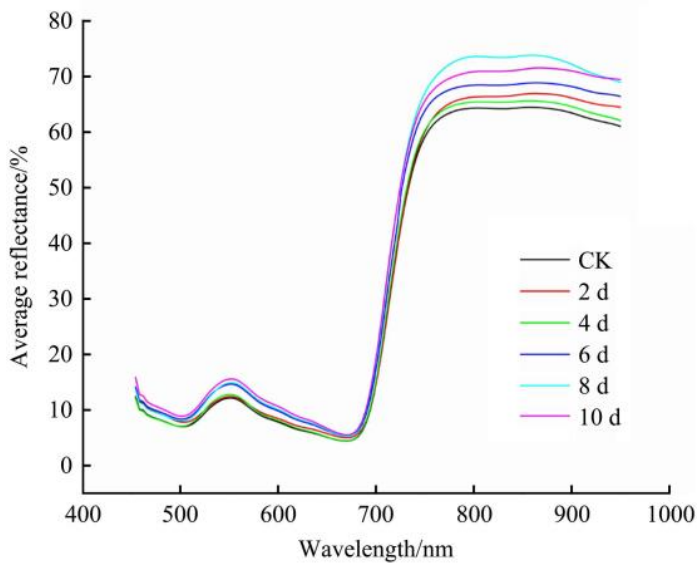

Figure 6 Average spectral curve of cotton leaves after the process of SG and MSC under different waterlogging conditions

\subsection{Detection of waterlogging stress}

The classification accuracy and loss function of GLNI-v3 and VGG-16 are shown in Figure 7 and Figure 8, respectively. Comparing the classification accuracy and loss values of the two models, it is found that the classification accuracy increases and the loss value decreases with the increasing number of training, for both the training and verification process of the two models. The average time for a round of training took $13.337 \mathrm{~s}$ for GLNI-v3 and 21.470 s for VGG-16. Compared with VGG-16, the classification accuracy of GLNI-v3 has a faster convergence rate. After 80 rounds of training, the verification accuracy of GLNI-v3 has reached $90 \%$, taking 18 minutes. VGG-16 took 54 minutes to reach $90 \%$ accuracy with 150 rounds of training. When the

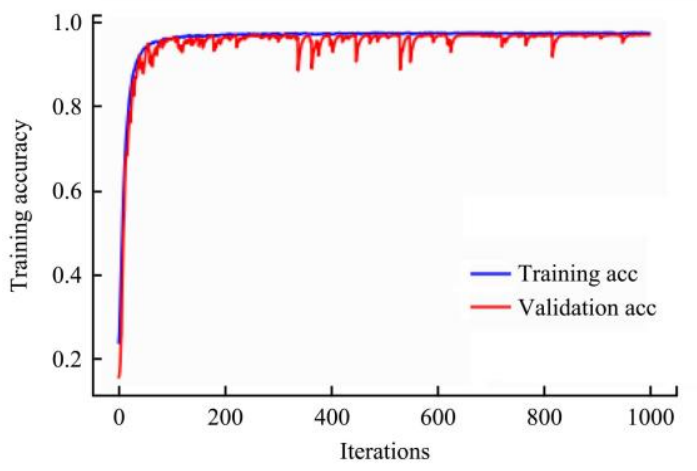

a. GLNI-v3 classification accuracy accuracy tends to remain almost unchanged, the loss of the value curve reaches the lowest point. The confusion matrix in Figure 9 shows 2 models has the same classification accuracy (97\%) for the verification set. GLNI-v3 had a lower loss value of 0.09431, whereas VGG-16 had a loss value of 0.17912. Both models had a good performance on classification of early waterlogging leaves, however, some leaves with $8 \mathrm{~d}$ waterlogging treatment were classified to $10 \mathrm{~d}$ waterlogging leaves erroneously. It could be the features after $8 \mathrm{~d}$ and $10 \mathrm{~d}$ waterlogging treatment were quite similar so that the deep learning models made mistake on the classification of long-term waterlogging leaves.

GLNI-v3 uses the Inception module to expand the width of the hidden layer of the convolutional neural network while maintaining a certain depth of the neural grid. VGG-16 uses only one size of convolution kernel to process input image in one convolutional layer. As the Inception module uses convolution kernels of different sizes to process the image at the same time, GLNI-v3 could obtain more image features in one training round under the same situation, and make classification accuracy converge faster.

Vegetation indices and multispectral images has been applied to estimate canopy cover and spray application rates of cotton by unmanned aerial vehicle in current researches ${ }^{[36,37]}$. With the day of waterlogging treatment increasing, it was found that spectrum of cotton changed greatly near $550 \mathrm{~nm}$ and $750 \mathrm{~nm}$, which could be regarded as the "green" and "red edge" band for a multispectral camera. These proves the possibility for multispectral images to detect waterlogging stress of cotton. RGB images are common materials for classification with deep learning ${ }^{[38-40]}$. This paper proved that hyperspectral images of cotton leaves with different waterlogging stress could be classified with CNN models. Considering the instruments of hyper-spectral imaging system are expensive and HIS cannot be acquired easily, multispectral images and RGB images may be adapted for the detection of waterlogging stress from the canopy of cotton on a large scale.

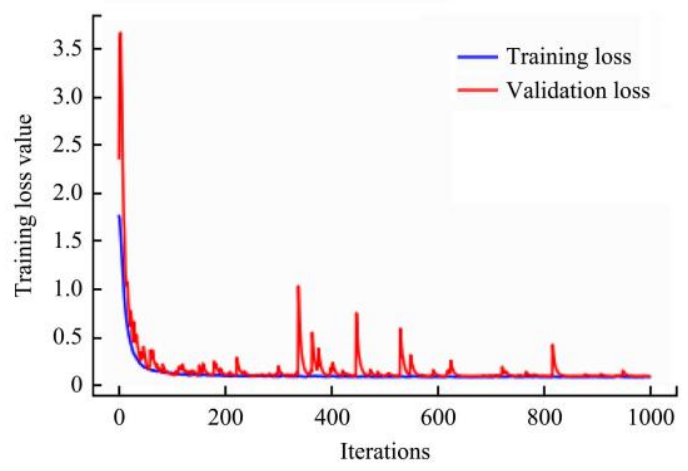

b. GLNI-v3 loss value

Figure 7 Training accuracy and loss value of GLNI-v3 model

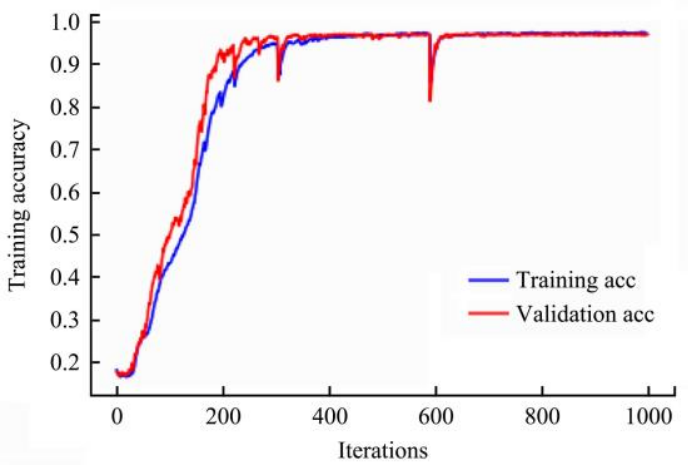

a. VGG-16 classification accuracy

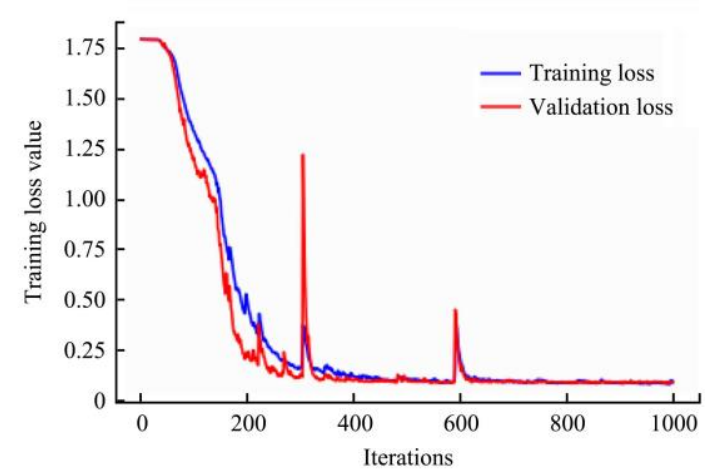

b. VGG-16 loss value

Figure 8 Training accuracy and loss value of VGG-16 model 


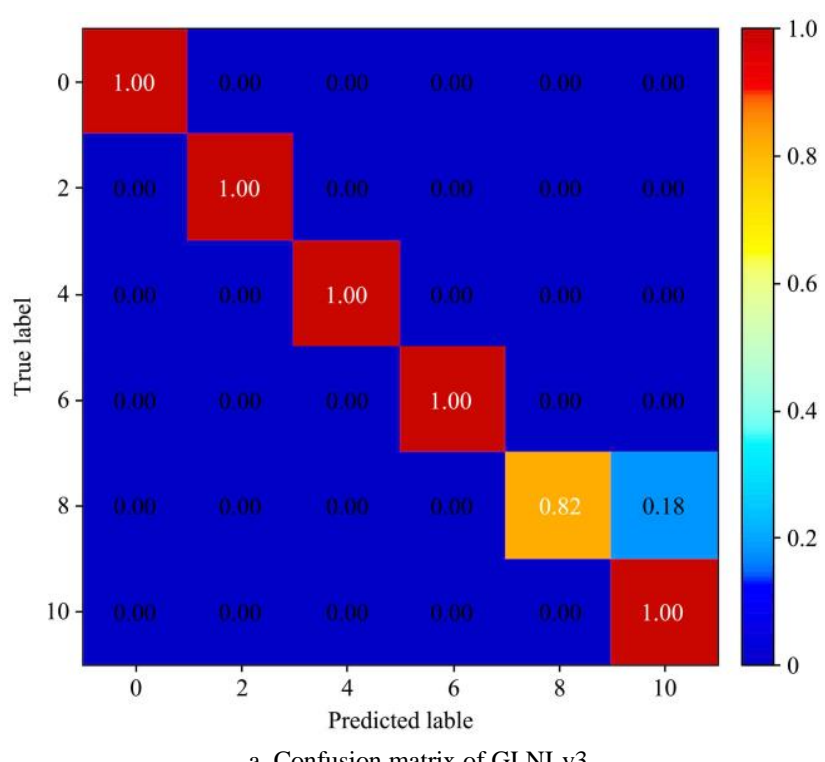

a. Confusion matrix of GLNI-v3

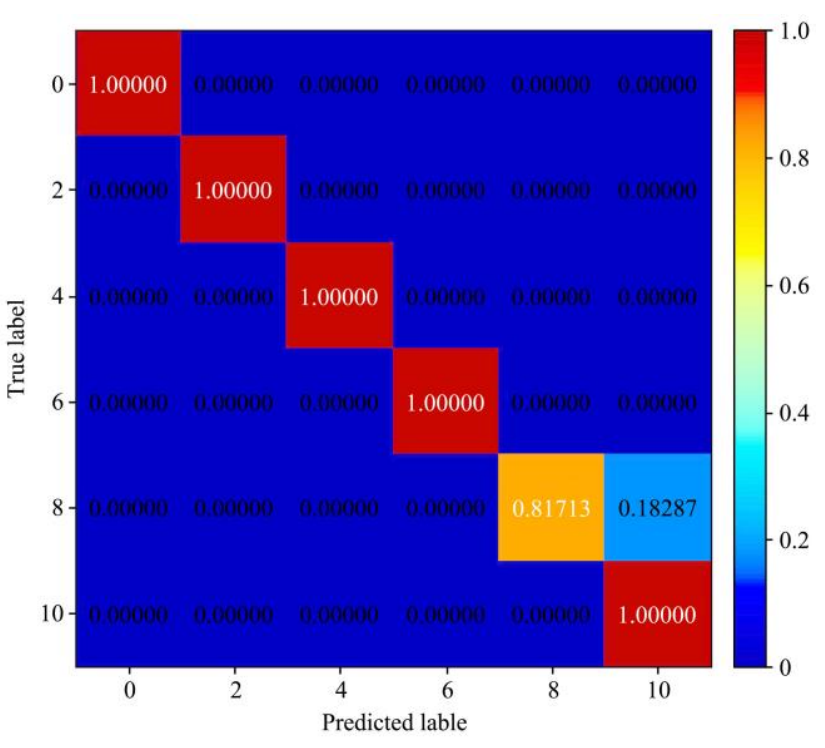

b. Confusion matrix of VGG-16

Note: 0 represents non-waterlogging treatment; 2 represents two days of waterlogging treatment; 4 represents four days of waterlogging treatment; 6 represents six days of waterlogging treatment; 8 represents eight days of waterlogging treatment; 10 represents ten days of waterlogging treatment.

Figure 9 Confusion matrix of classification results for GLNI-v3 and VG-16 models

\section{Conclusions}

Due to the climatic characteristics of the Yellow River Basin and the sensitive characteristics of cotton to waterlogging stress, the early detection of waterlogging stress provided a basis for the estimation of cotton yield loss and mitigation decision. The experiment with different days of waterlogging treatment was designed, and the HSIs of cotton leaves under waterlogging stress were obtained. Dimensionality reduction of image by principal component analysis was conducted, and convolutional neural network models were trained as classifiers for the detection of cotton waterlogging stress. The main conclusions are shown as follows:

(1) After SG smoothing and multiple scattering correction, the spectral curve of cotton leaves is similar to the original one, and the spectral curve is significantly different under different submergence days. With the increase of submergence days of cotton plants, the "green peak" of spectral curve increases gradually and becomes steeper near $550 \mathrm{~nm}$; in the range of "red edge" from 700 to $750 \mathrm{~nm}$, the beginning and end of the spectrum curve move forward, and there are 'blue shift' phenomena for the position of red edge of the spectra; in the platform of reflection peak from 750 to $950 \mathrm{~nm}$, the reflectance for the flooded cotton leaf is higher than that for the control group.

(2) The first principal component of hyperspectral image after dimensionality reduction was used to establish the data set, and the two classic convolutional neural network models of GLNI-v3 and VGG-16 were trained and tested. The experimental results showed that the accuracy of both GLNI-v3 and VGG-16 reached to 97\%; GLNI-v3 had a lower loss value of 0.09431 and took less time for training compared to VGG-16.

\section{Acknowledgements}

This study was supported by Top Talents Program for One Case One Discussion of Shandong Province and Agricultural Significant Application Technology Innovation Project of Shandong Province (Grant No. SD2019ZZ019).

\section{[References]}

[1] Hodgson A S, Chan K Y. The effect of short-term waterlogging during furrow irrigation of cotton in a cracking grey clay. Aust J Agric Res, 1982; 33(1): 109-116.

[2] Reicosky D C, Meyer W S, Schaefer N L, Sides R D. Cotton response to short-term waterlogging imposed with a water-table gradient facility. Agricultural Water Management, 1985; 10(2): 127-143.

[3] Hocking P J, Reicosky D C, Meyer W S. Effects of intermittent waterlogging on the mineral nutrition of cotton. Plant Soil, 1987; 101: 211-221.

[4] Bange M P, Milroy S P, Thongbai P. Growth and yield of cotton in response to waterlogging. Field Crop Res, 2004; 88(2-3): 129-142.

[5] Zhang Y J, Chen Y Z, Lu H Q, Kong X Q, Dai J L, Li Z H, et al. Growth, lint yield and changes in physiological attributes of cotton under temporal waterlogging. Field Crop Res, 2016; 194: 83-93.

[6] Milroy P S, Bange P M. Reduction in radiation use efficiency of cotton (Gossypium hirsutum L.) under repeated transient waterlogging in the field. Field Crop Res, 2013; 140: 51-58.

[7] Kuai J, Zhou Z G, Wang Y H, Meng Y L, Chen B L, Zhao W Q. The effects of short-term waterlogging on the lint yield and yield components of cotton with respect to boll position. Europ. J. Agronomy, 2015; 67: 61-74.

[8] Zhang Y J, Song X Z, Yang G Z, Li Z H, Lu H Q, Kong X Q, et al. Physiological and molecular adjustment of cotton to waterlogging at peak-flowering in relation to growth and yield. Field Crop Res, 2015; 179: 164-172.

[9] Milroy P S, Bange P M, Thongbai P. Cotton leaf nutrient concentrations in response to waterlogging under field conditions. Field Crop Res, 2009; 34: 246-255.

[10] Wang H M, Chen Y L, Hu W, Snider J L, Zhou Z G. Short-term soil-waterlogging contributes to cotton cross tolerance to chronic elevated temperature by regulating ROS metabolism in the subtending leaf. Plant Physiol Bioch, 2019; 139: 333-341.

[11] Najeeb U, Bange P M, Atwell B J, Tan D K Y. Understanding of the interactive effect of waterlogging and shade on cotton (Gossypium hirsutum L.) growth and yield. Procedia Environmental Sciences, 2015; 29: 85-86.

[12] Baranowski P, Jedryczka M, Mazurek W, Babula-Skowronska D, Siedliska A, Kaczmarek J. Hyperspectral and thermal imaging of oilseed rape (Brassica napus) response to fungal species of the genus alternaria. Plos One, 2015; 10(3): e0122913.

[13] Xia J A, Cao H X, Yang Y W, Zhang W X, Wan Q, Xu L, et al Detection of waterlogging stress based on hyperspectral images of oilseed rape leaves (Brassica napus L.). Comput Electron Agr, 2019; 159: 59-68.

[14] Moshou D, Pantazi X E, Kateris D, Gravalos I. Water stress detection 
based on optical multisensor fusion with a least squares support vector machine classifier. Biosyst Eng, 2014; 117: 15-22.

[15] Mahlein A K, Steiner U, Hillnhütter C, Dehne W H, Oerke E C. Hyperspectral imaging for small-scale analysis of symptoms caused by different sugar beet diseases. Plant Methods, 2012; 8(1): 3 .

[16] Gu Q, Sheng L, Zhang T H, Lu Y W, Zhang Z J, Zheng K F, et al. Early detection of tomato spotted wilt virus infection in tobacco using the hyperspectral imaging technique and machine learning algorithms. Comput Electron Agr, 2019; 167: 105066

[17] Xie C Q, Shao Y N, Li X L, He Y. Detection of early blight and late blight diseases on tomato leaves using hyperspectral imaging. Scientific Reports, 2015; 5: 16564

[18] Khan I H, Liu H Y, Cheng T, Tian Y C, Cao Q, Zhu Y, et al. Detection of wheat powdery mildew based on hyperspectral reflectance through SPA and PLS-LDA. Int J Precis Agric Aviat, 2020; 3(1): 13-24.

[19] Zhang M Y, Li C Y, Yang F Z. Classification of foreign matter embedded inside cotton lint using short wave infrared (SWIR) hyperspectral transmittance imaging. Comput Electron Agr, 2017; 139: 75-90.

[20] Zhang R Y, Li C Y, Zhang M Y, Rodgers J. Shortwave infrared hyperspectral reflectance imaging for cotton foreign matter classification. Comput Electron Agr, 2016; 127: 260-270.

[21] Prabhakar M, Prasad Y G, Thirupathi M, Sreedevi G, Dharajothi B, Venkateswarlu B. Use of ground based hyperspectral remote sensing for detection of stress in cotton caused by leafhopper (Hemiptera: Cicadellidae). Comput Electron Agr, 2011; 79: 189-198.

[22] Yang C H, Everitt J H, Fernandez C J. Comparison of airborne multispectral and hyperspectral imagery for mapping cotton root rot. Biosyst Eng, 2010; 107(2): 131-139.

[23] Yi Q X, Wang F M, Bao A M, Jiapaer G. Leaf and canopy water content estimation in cotton using hyperspectral indices and radiative transfer models. Int J Appl Earth Obs, 2014; 33: 67-75.

[24] Yi Q X, Bao A M, Wang Q, Zhao J. Estimation of leaf water content in cotton by means of hyperspectral indices. Comput Electron Agr, 2013; 90 : 144-151.

[25] Han X, Yu J Y, Lan Y B, Kong F X, Yi L L. Determination of application parameters for cotton defoliants in the Yellow River Basin. Int J Precis Agric Aviat, 2019; 2(1): 1-5.

[26] Schafer R W. What is a Savitzky-Golay filter? IEEE Signal Proc Mag, 2011; 28(4): 111-117.

[27] Chen J, Jonsson P, Tamura M, Gu Z H, Matsushita B, Eklundh L. A simple method for reconstructing a high quality NDVI time-series data set based on the Savitzky-Golay filter. Remote Sens Environ, 2004; 91(3-4):
332-344.

[28] Canny J. A computational approach to edge detection. IEEE T Pattern Anal, 1986; 8(6): 679-698.

[29] Liu Z Y, Wu F H, Huang J F. Application of neural networks to discriminate fungal infection levels in rice panicles using hyperspectral reflectance and principal components analysis. Comput Electron Agr, 2010; 72(2): 99-106

[30] Golzarian M R, Frick R A. Classification of images of wheat, ryegrass and brome grass species at early growth stages using principal component analysis. Plant Methods, 2011; 7(28): 1-11

[31] Szegedy C, Vanhoucke V, Ioffe S, Shlens J, Wojna Z. Rethinking the inception architecture for computer vision. arXiv: 1512.00567, 2016.

[32] LeCun Y, Bengio Y, Hinton G. Deep learning. Nature, 2015; 521: 436-444.

[33] Schmidhuber J. Deep learning in neural networks: an overview. Neura Networks, 2015; 61(1): 85-117.

[34] Abadi M, Agarwal A, Barham P, Brevdo E, Zhifeng C, Citro C, et al. TensorFlow: Large-scale machine learning on heterogeneous distributed systems. 2016. Available: https://blog.csdn.net/ynsshzwxhzyx/ article/details/79448631. Accessed on [2020-06-26].

[35] Simonyan K, Zisserman A. Very deep convolutional networks for large-scale image recognition. arXiv: 1409.1556, 2014.

[36] Akash A, Jinha J, Chang A J, Sungchan O, Murilo M, Juan L. A comparative study of RGB and multispectral sensor-based cotton canopy cover modelling using multi-temporal UAS data. Remote Sensing, 2019; 11(23): 2757. doi: 10.3390/rs11232757.

[37] Pedro H A M, Fabio H R B, Túlio H D M, João V P F F, Larissa P R T, Carlos A S J, et al. Estimating spray application rates in cotton using multispectral vegetation indices obtained using an unmanned aerial vehicle. Crop Protection, 2021; 40: 105407.

[38] Sungchan O, Chang A J, Akash A, Jinha J, Nothabo D, Murilo M, et al. Plant counting of cotton from UAS imagery using deep learning-based object detection framework. Remote Sensing, 2020; 12(18): 2981. doi : 10.3390/rs12182981.

[39] Feng A J, Zhou J F, Vories E, Sudduth K A. Evaluation of cotton emergence using UAV-based imagery and deep learning. Computers and Electronics in Agriculture, 2020; 177: 105711. doi: 10.1016/j.compag.2020.105711

[40] Adão N A, Witenberg S R S, Díbio L B. Cotton pests classification in field-based images using deep residual networks. Computers and Electronics in Agriculture, 2020; 174: 105488. doi: 10.1016/j.compag.2020.105488. 